University of Nebraska - Lincoln

DigitalCommons@University of Nebraska - Lincoln

Faculty Publications from the Department of Engineering Mechanics

Mechanical \& Materials Engineering,

Department of

April 2007

\title{
Calibration of atomic force microscope cantilevers using piezolevers
}

Saltuk B. Aksu

University of Nebraska - Lincoln

Joseph A. Turner

University of Nebraska - Lincoln, jaturner@unl.edu

Follow this and additional works at: https://digitalcommons.unl.edu/engineeringmechanicsfacpub

Part of the Mechanical Engineering Commons

Aksu, Saltuk B. and Turner, Joseph A., "Calibration of atomic force microscope cantilevers using piezolevers" (2007). Faculty Publications from the Department of Engineering Mechanics. 8. https://digitalcommons.unl.edu/engineeringmechanicsfacpub/8

This Article is brought to you for free and open access by the Mechanical \& Materials Engineering, Department of at DigitalCommons@University of Nebraska - Lincoln. It has been accepted for inclusion in Faculty Publications from the Department of Engineering Mechanics by an authorized administrator of DigitalCommons@University of Nebraska - Lincoln. 


\title{
Calibration of atomic force microscope cantilevers using piezolevers
}

\author{
Saltuk B. Aksu and Joseph A. Turner ${ }^{\text {) }}$ \\ Department of Engineering Mechanics, W317.4 Nebraska Hall, University of Nebraska-Lincoln, \\ Lincoln, Nebraska 68588-0526
}

(Received 23 August 2006; accepted 22 January 2007; published online 12 April 2007)

\begin{abstract}
The atomic force microscope (AFM) can provide qualitative information by numerous imaging modes, but it can also provide quantitative information when calibrated cantilevers are used. In this article a new technique is demonstrated to calibrate AFM cantilevers using a reference piezolever. Experiments are performed on 13 different commercially available cantilevers. The stiff cantilevers, whose stiffness is more than $0.4 \mathrm{~N} / \mathrm{m}$, are compared to the stiffness values measured using nanoindentation. The experimental data collected by the piezolever method is in good agreement with the nanoindentation data. Calibration with a piezolever is fast, easy, and nondestructive and a commercially available AFM is enough to perform the experiments. In addition, the AFM laser must not be calibrated. Calibration is reported here for cantilevers whose stiffness lies between 0.08 and 6.02 N/m. () 2007 American Institute of Physics. [DOI: 10.1063/1.2719649]
\end{abstract}

\section{INTRODUCTION}

Binning, Quate, and Gerber invented the atomic force microscope (AFM) in $1986 .{ }^{1}$ The AFM proved itself to be a powerful device to measure the topography of sample surfaces. In addition, quantitative measurements of interfacial forces can be made if calibrated cantilevers are used. ${ }^{2-4}$ This feature of the AFM became very useful in a variety of studies especially in biological applications. ${ }^{5-10}$ The geometry and material of the cantilever define both the overall mechanical properties of the cantilever, such as modulus of elasticity $E$, stiffness, and resonant frequency. Monocrystalline silicon and silicon nitride $\left(\mathrm{Si}_{3} \mathrm{~N}_{4}\right)$ are the most popular materials used for AFM cantilevers. Tungsten, nickel, and other materials are also used. ${ }^{11-17}$ Silicon nitride cantilevers can be produced thinner than other materials which gives them more flexibility and lower stiffness. For most applications, it is necessary to coat the backside of the cantilever with aluminum for better reflection of the laser. Although the coating is thin, its presence can complicate the understanding of the mechanical response. Rectangular thin bar and triangular lever forms are the most available geometries. The selection of the geometry and material of the cantilever depends on the objective of the experiment.

The force constant, usually reported in Newtons per meterN/m, defines the stiffness of a cantilever and relates tip force to tip displacement. Cantilevers with a stiffness $k$ less than $0.1 \mathrm{~N} / \mathrm{m}$ are designated as "soft" cantilevers ${ }^{18}$ and are mostly used in contact mode in order for the sample to remain undamaged during scanning. Cantilevers with $k$ higher than $1 \mathrm{~N} / \mathrm{m}$ are designated as "rigid" cantilevers and are mainly used in noncontact or dynamic modes as well as in atomic force acoustic microscopy. Their high stiffness leads to high resonant frequencies with small oscillation amplitudes. Although microfabrication processes have improved in

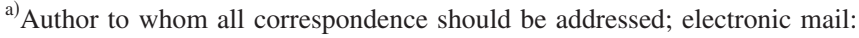
jaturner@unl.edu
}

the last decade, accurate control of AFM cantilever properties is still not possible. Precision AFM measurements require calibration of each cantilever used. Thus, fast and reliable calibration techniques are of high interest to the AFM community. In this article, a simple method for direct calibration of AFM cantilevers is presented that employs commercially available piezolevers. In the next section previous calibration methods are reviewed. In Sec. III, the experimental techniques are described. Experimental results are presented and discussed in Sec. IV and conclusions are presented in Sec. V.

\section{CURRENT CANTILEVER CALIBRATION METHODS}

The AFM can provide qualitative information by numerous imaging modes but it can also provide quantitative information if calibrated cantilevers are used. When measuring interaction forces between the sample and the AFM tip, an accurate value of the AFM cantilever stiffness is needed. Therefore, the ability to calibrate the cantilevers used in AFM is of fundamental importance. To date, several techniques have been developed to determine the spring constant of the AFM cantilevers. These techniques can be divided into three main categories: theoretical techniques, static response techniques, and dynamic techniques.

\section{A. Theoretical techniques}

Theoretical techniques are based on the prediction of cantilever spring constant from geometric information alone. They use small deflection theories usually assuming that the cantilever behaves as a linearly elastic and isotropic material. ${ }^{19}$ These methods employ different formulas for different geometries of cantilevers and rely on precise knowledge of the geometry and the modulus of elasticity of the cantilever. $^{20,21}$ Rectangular and V-shaped cantilevers are the most widely used geometries. Due to manufacturing processes, the dimensions of cantilevers, especially the thickness, are difficult to control along the length of the cantilever 
and may even vary for each individual cantilever on the same chip. Thus, every measurement of the dimensions of the cantilever has some uncertainty. The uncertainty of the thickness has a profound effect on the overall theoretical stiffness uncertainty. Also, tip coatings are used in some special applications and these coatings can have a dramatic effect on the stiffness. ${ }^{22}$ Thus, theoretical techniques without any experimental input are not very reliable.

\section{B. Static response techniques}

Static response techniques focus on the deflection of the cantilever when a known force is applied to the AFM cantilever. The known force may be applied either with a pendulum, ${ }^{23}$ an added mass, ${ }^{24}$ or with a precalibrated cantilever. ${ }^{3,25-28}$

In static response to pendulum force method, a specified force is applied to the AFM cantilever by a precalibrated pendulum. The horizontal displacement of the mass of the pendulum and the AFM cantilevers are measured. Then the stiffness of the AFM cantilever is calculated with the formula ${ }^{19}$

$$
k_{z}=\frac{M_{p} g}{L_{p}} \frac{\Delta z_{p}}{\Delta z_{c}},
$$

where $M_{p}$ is the mass of the pendulum, $g$ is the gravitational acceleration, $\Delta z_{p}$ is the horizontal displacement of the pendulum, $L_{p}$ is the length of the pendulum, and $\Delta z_{c}$ is the horizontal displacement of the AFM cantilever. This method requires the AFM cantilever to be positioned vertically with the pendulum mass, which is not possible in most commercially available AFMs. The accuracy of the experiment decays with the increase in the difference of the AFM cantilever spring constant and the pendulum spring constant. Therefore, a prior estimate of the stiffness of the cantilever is needed and calibration of the AFM laser reflection is required. This method is time consuming and lacks accuracy. The errors of this method are reported to be as high as $50 \% .^{19}$

Cantilever on cantilever methods require micromanipulation and precise aligning of the AFM cantilever on the reference cantilever. If calibration of the AFM laser is not performed, an optical microscope may be utilized while applying a known force to the cantilever. The AFM stage must be calibrated in order to know the total displacement of the reference cantilever. The deflection of the cantilever is then measured and the stiffness is calculated using simple beam theory. As in the pendulum method the reference cantilever stiffness must be chosen appropriately. These methods can be applied to arbitrarily shaped and coated cantilevers.

Another version of the static response methods is the added mass method. The first step for static deflection with added mass method is the attachment of a calibrated mass to the end point of the AFM cantilever-a technique that is potentially destructive. Then the deflection of the cantilever is measured. The magnitude of the mass and the location of the mass must be measured precisely in order to obtain accurate results. Then the mass is removed. Despite its drawbacks, this method does not require a prior estimate of the stiffness of the AFM cantilever.

\section{Dynamic response methods}

Dynamic response methods such as the dynamic added mass resonance method, ${ }^{29}$ thermal noise method, ${ }^{30}$ unloaded frequency method, ${ }^{31}$ and modified unloaded frequency method, ${ }^{32}$ use the resonant frequencies combined with the geometrical information of the cantilever to determine the spring constant. Thus, these methods are indirect: The measured quantity is neither force nor displacement such that a conversion for spring constant is necessary. In order to obtain good results from the dynamic added mass resonance method, experiments should be repeated with different spheres. This condition makes this method potentially destructive and difficult to implement. The sensitivity of the device used to measure the noise in the deflection signal determines the limits of the thermal noise method. This method is used for the cantilevers whose stiffness is less than $1 \mathrm{~N} / \mathrm{m}$. It is nondestructive and can be applied to arbitrarily shaped and coated cantilevers. However, inversion of the measured frequency response requires assumptions about the cantilever (material, geometry, etc.) that are difficult to corroborate. In the unloaded frequency method the stiffness of the AFM cantilever is obtained by a single measurement of the first resonant frequency of the cantilever in vacuum. The measurement of the mass of the cantilever, required for determining its spring constant, is not usually specified by the manufacturer. This method is especially difficult for coated cantilevers and cantilevers which are not composed of single materials. However, these drawbacks are often outweighed by the fact that an added mass is not needed.

Other methods where specific instruments are utilized, such as a nanoindenter, ${ }^{33}$ piezolever, ${ }^{34,35}$ or a microfabricated array of reference spring (MARS) device, ${ }^{36}$ require specific equipment and significant experimental setup. The nanoindentation method is fast and simple, but precise positioning of the indenter tip on the cantilever is required. This method is currently not applicable to soft cantilevers due to load limits of current indentation instruments. Although the MARS device method involves significant AFM cantilever stiffness constraints (must be between 0.01 and $4 \mathrm{~N} / \mathrm{m}$ ), and is not yet commercially available, it seems to be one of the best calibration methods because it provides a direct measure of the force applied and the resulting displacement.

It is clear that each calibration method has issues associated with practicality. Thus, choosing a calibration method for a specific cantilever is not an easy task. The accuracy, applicability, simplicity, and duration of the procedure specify the method to be used. The methods reviewed here are summarized in Table I. Previous results highlight the utility of a method for which both force and displacement are measured directly.

\section{CALIBRATION USING A PIEZOLEVER}

In this article, a commercially available Si self-sensing piezoresistive cantilever (piezolever) was used to calibrate 13 different commercially available cantilevers. The piezolever (PRC400:SII Nanotechnology Inc.) ${ }^{37}$ was first calibrated using a Triboscope nanoindentation device (Hysitron Inc.). The piezolever was subsequently used to calibrate the 
TABLE I. Comparison of the calibration methods for AFM cantilevers.

\begin{tabular}{|c|c|c|c|c|c|}
\hline Method and Accuracy & Class & $\begin{array}{l}\text { Prior estimate } \\
\quad \text { of } k\end{array}$ & $\begin{array}{l}\text { Potentially } \\
\text { destructive }\end{array}$ & $\begin{array}{l}\text { Arbitrary shaped } \\
\text { and coated } \\
\text { cantilevers }\end{array}$ & $\begin{array}{l}\text { A prior calibration } \\
\text { of the AFM laser } \\
\text { reflection is needed }\end{array}$ \\
\hline Finite difference (Ref. 21), \%10+ (Ref. 36) & Theoretical & Not required & No & Not applicable & No \\
\hline Parallel beam calculation (Refs. 31 and 24), \%10+ (Ref. 36) & Theoretical & Not required & No & Not applicable & No \\
\hline $\begin{array}{l}\text { Static response to pendulum force (Ref. 23), \%30-50 (Refs. } 33 \\
\text { and 19) }\end{array}$ & Static & Required & No & Applicable & Yes \\
\hline Static deflection with added mass (Ref. 24), \%20 (Ref. 19) & Static & Required & Yes & Applicable & Yes \\
\hline $\begin{array}{l}\text { Static response with a calibrated cantilever (Refs. 27, 3, and 28), } \\
\% 20 \text { (Ref. 19) }\end{array}$ & Static & Required & No & Applicable & Yes \\
\hline Dynamic added mass resonance (Ref. 29), \%10 (Refs. 19 and 33) & Dynamic & Not required & Yes & Applicable & No \\
\hline Thermal noise (Ref. 30), \%20 (Ref. 19) & Dynamic & Required & No & Applicable & Yes \\
\hline Unloaded frequency (Ref. 31), \%10 (Ref. 19) & Dynamic & Not required & No & Not applicable & No \\
\hline Modified Unloaded frequency (Ref. 32), \%10 (Ref. 19) & Dynamic & Not required & No & Applicable & No \\
\hline $\begin{array}{l}\text { Calibration with Microfabricated Array of Reference Springs } \\
\text { (Mars Device) (Ref. 36) }\end{array}$ & Other & Required & No & Applicable & No \\
\hline Nanoindentation (Ref. 33), \%10 (Ref. 33) & Other & Required & No & Applicable & No \\
\hline
\end{tabular}

AFM stage. Finally, the various AFM cantilevers were calibrated. The use of a piezolever for calibration is a variation on the static response with a calibrated cantilever method with a couple of important differences. In the usual methods, the reference cantilever is placed in contact with the AFM cantilever, and the deflections of both cantilevers are measured indirectly using either optical microscopes or a combination of optical microscopes and the AFM itself. Unless the calibration cantilever is calibrated by the user, the experimental accuracy is based upon the stiffness value of the reference cantilever provided by the manufacturer. In Tortonese et al. ${ }^{28}$ the stiffness of the calibration cantilever is calculated by the formula

$$
k=58.8 \rho \sqrt{(\rho / E)} w L^{3} F_{r}^{3},
$$

where $\rho$ is the density of the cantilever material, $E$ is the modulus of elasticity, $L$ is the length of the cantilever, $w$ is the width, and $F_{r}$ is the resonant frequency of the cantilever in vacuum. Then this cantilever is used to calibrate the sample AFM cantilevers. Thus, the frequency of the calibration cantilever must be measured. However, it is a common practice for the manufacturer to measure the resonant frequencies of only a few cantilevers from a given wafer and use the average value for all the cantilevers belonging to that particular wafer. Also, instead of the measured values of $L$ and $w$, the nominal values are often used and the thickness must be assumed constant along their length. As mentioned before, the dimensions of cantilevers, especially the thickness, are difficult to control along the length of the cantilever and may vary even for individual cantilevers on the same chip. The fact that second or third resonances often do not scale relative to the first according to simple beam theories highlights the potential error of these indirect methods. Therefore, the calibration cantilevers must be calibrated with a direct method before they can be used as reference cantilevers.

In Gibson et al., ${ }^{3}$ the reference cantilever is calibrated using simple beam theory

$$
k=\frac{E w t^{3}}{4 L^{3}},
$$

where $t$ is the thickness of the cantilever. The dimensions of the cantilever are measured by scanning electron microscope (SEM). The thickness of the cantilever is again assumed to be constant along the cantilever. In Torii et al. ${ }^{27}$ the reference cantilever is calibrated by the static mass method which is reported to have up to a $20 \%$ error. ${ }^{19}$

However, with the piezolever method bias error is reduced by calibrating the relationship between applied force, deflection, and output voltage of the piezolever using nanoindentation. This feature of the piezolever allows calibration of AFM cantilevers without any complicated deflection measurements once the piezolever and the AFM stage are calibrated. The piezolever has two sensing resistors. As shown in Fig. 1, these two resistors are placed in a Wheatstone bridge and the deflection of the piezolever corresponds to changes in voltage. The specifications of the piezolever that was used in the experiments here are given in Table II. Calibration of the piezolever using nanoindentation is described in part A, while calibration of the AFM stage using the calibrated piezolever is explained in part B. Part C is a detailed description of the proposed cantilever calibration technique used to calibrate AFM cantilevers.

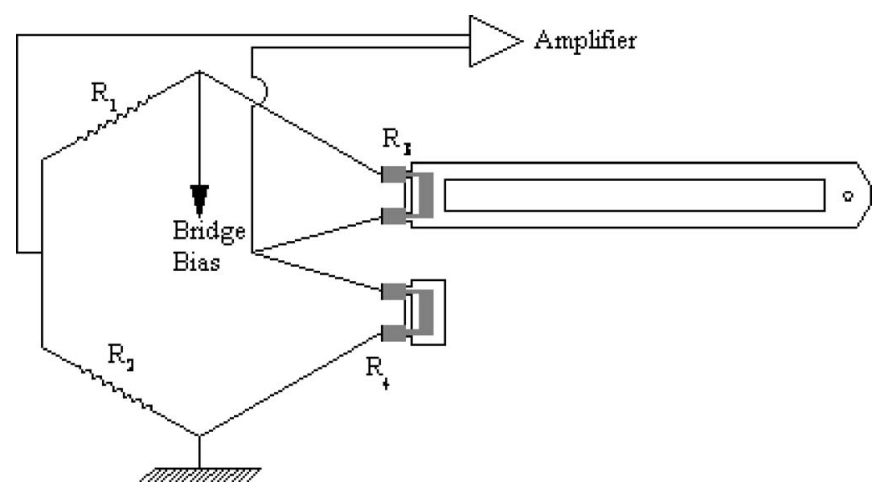

FIG. 1. Wheatstone bridge for the piezolever. 
TABLE II. Nominal specifications of the piezolever.

\begin{tabular}{cc}
\hline \hline Cantilever & PRC400 \\
\hline Length $(\mu \mathrm{m})$ & 400 \\
Thickness $(\mu \mathrm{m})$ & $4-5$ \\
Width $(\mu \mathrm{m})$ & 50 \\
Force constant $(\mathrm{N} / \mathrm{m})$ & $2-4$ \\
Tip radius $(\mathrm{nm})$ & $20-50$ \\
Tip height $(\mu \mathrm{m})$ & $6-8$ \\
Measured stiffness $(\mathrm{N} / \mathrm{m})$ & 3.4 \\
\hline \hline
\end{tabular}

\section{A. Piezolever calibration}

Nanoindentation tests are perhaps the most common tests for measuring the mechanical properties of materials at small scales. ${ }^{38}$ A number of commercial instruments for nanoindentation experiments are currently available. These methods allow the load applied to the sample as well as the tip displacement to be recorded. The contact area and mechanical properties are obtained by analyzing the data recorded using appropriate contact mechanics theories. For the Triboscope, the electrostatic force generated between the center plate and one of the fixed plates applies the load to the center plate. Using the two capacitances formed by the three parallel plates, the applied load and the nanoindentation depth can be measured. Because this instrument gives the force versus time and the depth displacement versus time information simultaneously, it can be effectively used to deflect AFM cantilevers. However, the nanoindentation method is not applicable to very soft AFM cantilevers due to current load limitations. The sensitivity of the nanoindentation instrument is determined by the force noise floor. The force noise floor is the standard deviation of the force signal taken in air. It is the lowest force that can be measured given the electronic and physical noise that affect the measurements. Theoretically, the most compliant cantilever that could be tested using nanoindentation is given by (force noise floor $\times 2$ )/maximum displacement. Thus, for the Hysitron Triboscope used here, the minimum stiffness value is $0.4 \mathrm{~N} / \mathrm{m}$.

The steps of the procedure to calibrate the piezolever are summarized in Fig. 2. The AFM cantilever is located under the indenter tip [Fig. 2(a)]. The indenter tip is carefully brought within microns of the AFM tip using optical views [Fig. 2(b)]. Using the indenter software the indenter tip is engaged with the AFM cantilever and an indent is performed [Fig. 2(c)]. Here, a trapezoidal indent is used. [Fig. 2(d)]. The trapezoidal indent takes $35 \mathrm{~s}$ and consists of three parts. The first part is the loading zone-the load is applied to the cantilever starting from the set point to the maximum value linearly in $5 \mathrm{~s}$. The second part applies the constant maximum force for $25 \mathrm{~s}$. This duration allows a stable voltage readout from the piezolever. The third part is the relaxation part- the load is reduced to the setpoint linearly in $5 \mathrm{~s}$. This procedure is repeated ten times with different maximum values.

After the data are collected, a load-depth curve is displayed from which the stiffness of the AFM cantilever is extracted. The deformation of the cantilever for a typical load range is elastic, such that the data may be fit with a linear regression. As seen in Fig. 3, the force constant of the

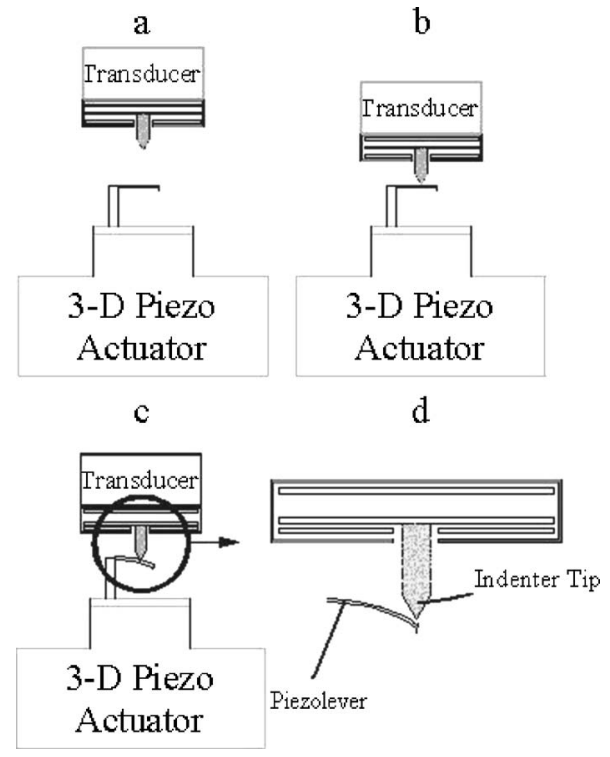

FIG. 2. Calibration using nanoindentation. (a) The AFM cantilever is located under the indenter tip. (b) The indenter tip is carefully brought within microns of the AFM tip using optical views. (c) Using the indenter software the indenter tip is engaged with the AFM cantilever and an indent is performed. (d) The desired stiffness value is obtained by an indent at the end of the cantilever, such that an indent is performed at the end of the AFM cantilever.

cantilever is obtained from the slope of the force-distance curve. This type of actuation has good temperature stability and small size of system. On the other hand its displacement resolution is limited to tenths of microns and the load range is limited to tens of micronewtons. The biggest challenge with this technique is the alignment of the indenter tip with the probe tip. The difficulty arises from the fact that optical views from two perpendicular directions are required in order to align the indenter tip in both longitudinal and lateral directions. Here, the nanoindentation method is used to calibrate the piezolever such that the measured stiffness value of the piezolever is very reliable. With the piezolever calibrated in this way, it may then be used for subsequent measurements.

\section{B. Calibration of the AFM stage}

The AFM stage must first be calibrated in order to measure the stage displacement precisely. It is a common prac-

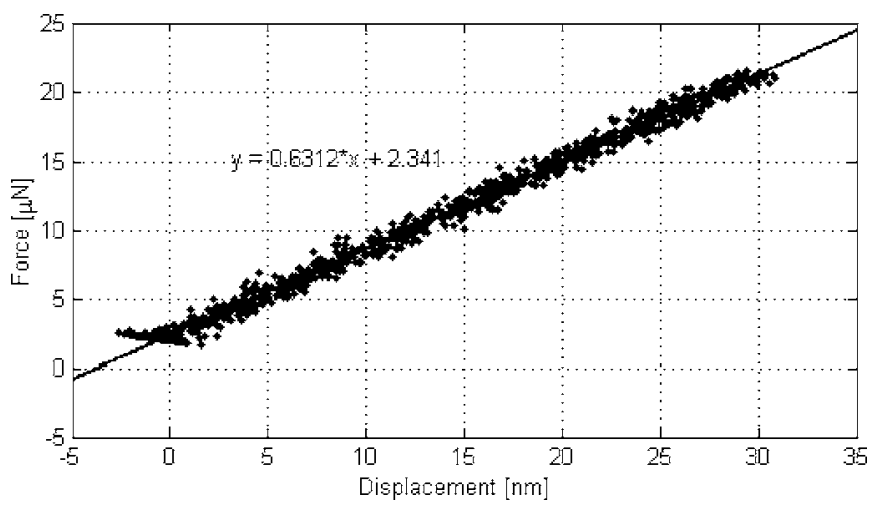

FIG. 3. Typical force-displacement data collected for an AFM cantilever using nanoindentation with the linear curve fit. 


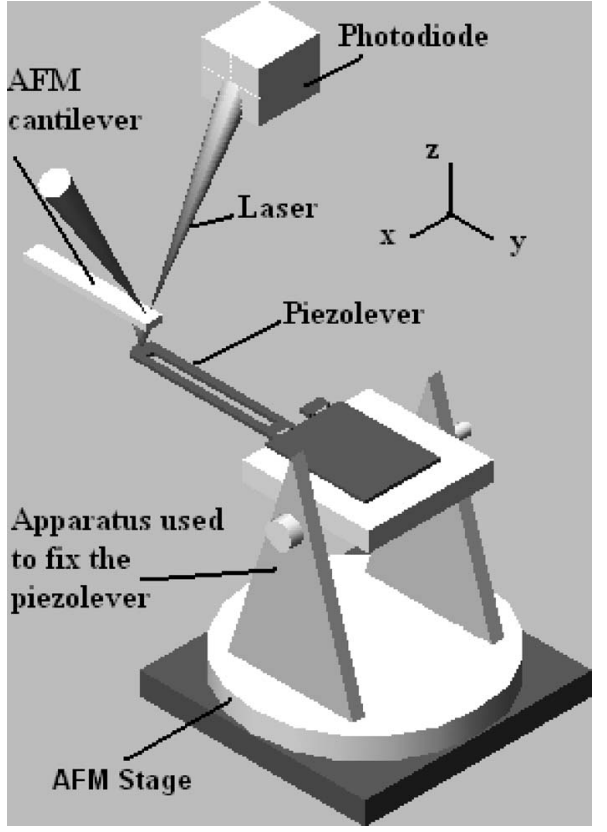

FIG. 4. Experimental setup for the calibration using the piezolever method. An apparatus is designed to fix the piezolever under the AFM cantilever. The piezolever is then placed in contact with the AFM cantilever.

tice to calibrate the stage using the method described in the user manual of the AFM. ${ }^{39}$ In this case, an image of a reference grating sample is obtained using the AFM and the height of the sample is extracted from the image. The stage is then calibrated by multiplying the existing calibration constant with an appropriate correction factor. The height of the grating which can be used for $z$-axis calibration is typically hundreds of nanometers. Then in order to convert the photodiode output signal to deflection, the response of the photosensor must be calibrated. In the proposed method, this process is not necessary. Although this technique is sufficient for small displacements, it is not applicable for larger displacements. Thus, nonlinear stage behavior over the entire range of stage movement ( $8 \mu \mathrm{m}$ in this case) cannot be assessed. Here, a calibrated piezolever was used to calibrate the stage using a cantilever chip. After engaging the piezolever, the feedback mechanism was disabled and, using the software of the AFM, the stage was raised using $0.25 \mu \mathrm{m}$ steps. Since the cantilever chip directly contacted the end of the piezolever, this method guarantees that all piezolever displacement corresponds to motion at the end of the piezolever. A total of $8 \mu \mathrm{m}$ displacement in the $z$ axis was swept using this technique and the voltage response of the piezolever was recorded. The overall response was fit with a quadratic function that captured the nonlinear AFM stage response well.
The measured calibration factor of the stage is then used for calibration of AFM cantilevers.

\section{Calibration of AFM cantilevers}

The calibration of AFM cantilevers can be summarized as follows. The designed apparatus (shown in Fig. 4) is placed on the AFM stage (the height of the apparatus is 13 $\mathrm{mm}$ and can be placed on all commercially available AFMs easily). The piezolever is located using the optical microscope of the AFM [Fig. 5(a)]. The uncalibrated AFM cantilever is brought very close to the piezolever with the $z$ control of the AFM [Fig. 5(b)] and the AFM cantilever is aligned directly above the piezolever. Then, using the AFM software, the cantilevers are brought into contact. This procedure is repeated until the AFM cantilever tip is in contact with the reverse side of the piezolever tip [Fig. 5(c)]. Then, the voltage output from the piezolever is reset to zero. The alignment of the AFM cantilever is very important because it may be a major error source (discussed below). Ideally the AFM cantilever should be in contact with the exact location where the indenter tip contacted previously for calibration of the piezolever itself. Thus, extra care should be taken for the alignment of the cantilevers.

After a successful contact is made, the base of the piezolever is moved up in the $+z$ direction using the manual control of the AFM stage (Fig. 6) and the change in voltage is recorded. Since the AFM stage is calibrated previously using the piezolever itself, the precise displacement of the base is known. This process is repeated until sufficient data are collected. Each motion of the AFM stage in the $+z$ direction causes deflection of both the AFM cantilever and the piezolever. Assuming the contact of the cantilevers is within a couple of micrometers of the center, there are no contributions from the friction forces and there is no penetration of the AFM cantilever tip on the piezolever, the relationship between the total displacement of the stage and the deflections of the cantilevers is given by

$$
\delta_{T}=\delta_{C}+\delta_{P},
$$

where $\delta_{T}$ is the total displacement of the stage, $\delta_{C}$ is the deflection of the AFM cantilever, and $\delta_{P}$ is the deflection of the piezolever. In addition to the piezolever stiffness, two of these three parameters must be known to calibrate the AFM cantilever. In the proposed method, $\delta_{T}$ and $\delta_{P}$ are known due to the procedure explained above. The stiffness of the AFM cantilever $k_{C}$ then can be written in the form ${ }^{28}$

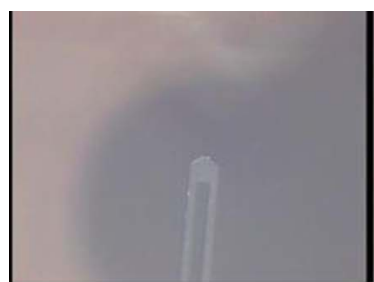

a)

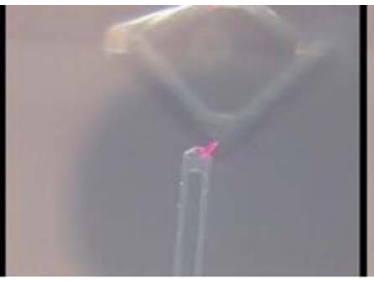

b)

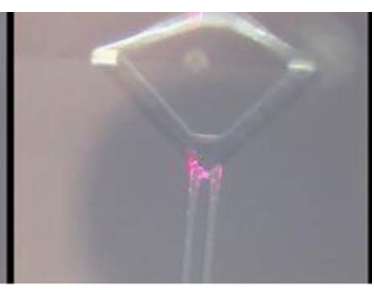

c)
FIG. 5. Calibration methodology using piezolever. (a) The piezolever is located using the optical microscope of the AFM. (b) The uncalibrated AFM cantilever is brought very close to the piezolever with the $z$ control of the AFM. (c) The AFM cantilever is aligned directly above the piezolever and using the AFM software, the cantilevers are brought into contact. 


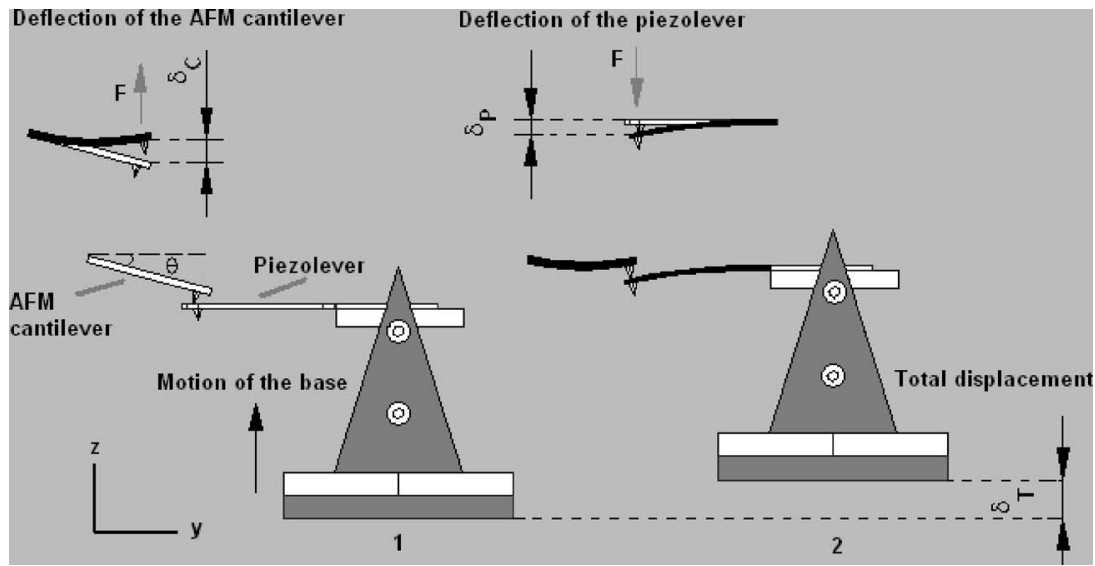

FIG. 6. Schematic of the calibration procedure of the AFM cantilevers using a piezolever. (1) The AFM cantilever is placed into contact with the piezolever. (2) The AFM stage is moved in the $+\mathrm{z}$ direction while the AFM cantilever is kept in its original location. The deflection of the stage $\delta_{T}$ is controlled by the AFM software. The deflection of the piezolever $\delta_{P}$ is extracted by the voltage change of the piezolever. The displacement of the AFM cantilever $\delta_{C}$ is calculated by using Eq. (4).

$$
k_{C}=k_{P} \frac{\delta_{P}}{\delta_{C} \cos (\theta)},
$$

where $\theta$ is the angle between the AFM cantilever and the horizontal $x$ axis before the deflection. Since the total displacement of the AFM stage is known and the force displacement relationship of the piezolever can be calculated, the force-displacement response of the AFM cantilever is determined. A linear curve is fit to the force-displacement data, from which the slope of the curve defines the AFM spring constant.

\section{EXPERIMENTAL RESULTS}

The measurements described above are used to calibrate 13 commercially available cantilevers. The experimental results are summarized in Table III. Six triangular and seven rectangular cantilevers are calibrated using both nanoindentation and the piezolever method. Since the nanoindentation method is not applicable for cantilevers whose stiffness is less than $0.4 \mathrm{~N} / \mathrm{m}$, nanoindentation was not performed on cantilevers 11, 12, and 13. An example of data collected using the nanoindentation method is shown in Fig. 3. As seen from Table III, nanoindentation results for cantilevers 5 and 6 , whose nominal stiffness values are between 1.5 and $5 \mathrm{~N} / \mathrm{m}$ are not reliable. The stiffness of three of the cantilevers was measured as $0.5 \mathrm{~N} / \mathrm{m}$ using the piezolever method but the results for the same cantilevers differ from the nanoindentation measurements. These results suggest that nanoindentation may give poor results for the cantilevers whose stiffness is between 0.4 and $1 \mathrm{~N} / \mathrm{m} .{ }^{33}$ For the cantilevers whose stiffness is higher than $1 \mathrm{~N} / \mathrm{m}$, the results from nanoindentation are in a very good agreement with the results from the piezolever. Ideally, the stiffness values of all cantilevers are expected to fall within the nominal stiffness range provided by the manufacturer. However, the stiffness values measured using these two different experiments show that out of 13 cantilevers, only four cantilevers fall within the nominal stiffness range given by the manufacturer. Thus, these data make clear the primary motivation for these experiments.

The accuracy of the nanoindentation method is determined by calculating the standard deviation of the indentation data, whereas the accuracy of the piezolever method is a combination of the standard deviation of the piezolever experimental data and the tolerance of the voltmeter. The accuracy of the piezolever method is seen to be higher than the nanoindentation method for the 12 cantilevers. The errors in these experiments can be summarized as instrumentation errors, random errors, and errors caused by misalignment. The instrument constants used for the nanoindentation are given in Table IV. ${ }^{40}$ Among these constants, only the electrostatic force constant (EFC) is tuned; the others are not changed. The mechanical resistance of the springs integrated to the

TABLE III. Experimentally measured stiffness values of two types of cantilevers are compared to the nominal values supplied by the manufacturer. Nanoindentation and the proposed piezolever method are used for the measurements.

\begin{tabular}{|c|c|c|c|c|}
\hline & \multirow[b]{2}{*}{ Cantilever } & \multicolumn{3}{|c|}{ Cantilever spring constant, $k_{C}(\mathrm{~N} / \mathrm{m})$} \\
\hline & & Nominal value & Nanoindentation & Piezolever \\
\hline 1 & Ultrasharp NSC11/Si3N4/ALBS type A & $1.5-5$ (Ref. 41) & $0.2 \pm 0.64$ & $0.57 \pm 0.12$ \\
\hline 2 & Ultrasharp NSC11/Si3N4/ALBS type A & $1.5-5$ (Ref. 41) & $2.1 \pm 0.59$ & $2.05 \pm 0.47$ \\
\hline 3 & Ultrasharp NSC11/Si3N4/ALBS type A & $1.5-5$ (Ref. 41) & $1.1 \pm 0.46$ & $0.94 \pm 0.16$ \\
\hline 4 & Ultrasharp NSC11/Si3N4/ALBS type A & $1.5-5$ (Ref. 41$)$ & $0.2 \pm 0.59$ & $0.49 \pm 0.03$ \\
\hline 5 & Ultrasharp NSC11/Si3N4/ALBS type A & $1.5-5$ (Ref. 41$)$ & $0.5 \pm 0.68$ & $0.51 \pm 0.1$ \\
\hline 6 & Ultrasharp NSC11/Si3N4/ALBS type A & $1.5-5$ (Ref. 41) & $1.1 \pm 0.65$ & $0.94 \pm 0.13$ \\
\hline 7 & Rectangular cantilever $200 \mu \mathrm{m}$ length & $2.3 \pm 0.23$ & $1.2 \pm 0.65$ & $0.97 \pm 0.36$ \\
\hline 8 & Rectangular cantilever $200 \mu \mathrm{m}$ length & $2.3 \pm 0.23$ & $1.5 \pm 0.65$ & $1.02 \pm 0.43$ \\
\hline 9 & Rectangular cantilever $200 \mu \mathrm{m}$ length & $2.3 \pm 0.23$ & $1.3 \pm 0.69$ & $1.08 \pm 0.41$ \\
\hline 10 & Rectangular cantilever $400 \mu \mathrm{m}$ length & $0.3 \pm 0.03$ & N/A & $0.17 \pm 0.07$ \\
\hline 11 & Rectangular cantilever $400 \mu \mathrm{m}$ length & $0.3 \pm 0.03$ & N/A & $0.13 \pm 0.1$ \\
\hline 12 & Rectangular cantilever $400 \mu \mathrm{m}$ length & $0.3 \pm 0.03$ & N/A & $0.16 \pm 0.06$ \\
\hline 13 & Rectangular cantilever $400 \mu \mathrm{m}$ length & $0.3 \pm 0.03$ & N/A & $0.16 \pm 0.07$ \\
\hline
\end{tabular}


TABLE IV. Instrumentation constants used in nanoindentation measurements.

\begin{tabular}{ccc}
\hline \hline Constant & Value & Unit \\
\hline Tare & -306 & $\mathrm{Mg}$ \\
Load scale factor (force) & 1 & $\mathrm{mV} / \mathrm{mg}$ \\
Displacement scale factor (deflection) & 20.411 & $\mathrm{mV} / \mu \mathrm{m}$ \\
Electrostatic force constant & 0.0239 & $\mu \mathrm{N} / \mathrm{V}^{2}$ \\
Self calibration check & 473 & $\mathrm{mg}$ \\
Maximum force & 8.604 & $\mathrm{mN}$ \\
\hline \hline
\end{tabular}

plates in the Triboscope are compensated with the EFC, which is the major source of bias error. When a new tip is installed an air indent allows the EFC to be adjusted. During the indent the middle plate moves freely against the springs holding it. Once the Triboscope is calibrated, the Hysitron software compensates these spring forces by subtracting them from the actual indent forces. Therefore, the force displacement curve of the actual indent remains. The EFC is calibrated before starting indentation and periodically measured during the experiments in order to minimize the errors related to instrumentation. Random errors mainly arise from vibrations in the apparatus, electrical fluctuations, and many other small but uncontrolled effects. In an ideal environment the signal measured by the transducer is very clean, and there is almost no noise, allowing a very high sensitivity. In reality there is some noise which may come from external sources, such as electromagnetic interference from nearby equipment and transmission lines, and from internally generated noise on the cabling. Random errors add additional noise to the signal measured by the transducer and increase the minimum stiffness which can be measured. These errors were minimized by allowing the instruments to warm up for one hour before the experiments. In addition, all experiments were performed under the same ambient room conditions.

As mentioned earlier, in the nanoindentation method, the most important part of the experiment is the alignment of the tip with the AFM cantilever. In an ideal case the indenter tip should touch the point on the cantilever exactly above the AFM probe tip. Misalignment errors can be either in the lateral direction or the normal direction. The cantilevers should be aligned very carefully in both of the experiments. Since lateral misalignment causes direct errors in the stiffness measurement, great care must be taken to ensure that the alignment is performed correctly.

\section{SUMMARY}

In this article, a compact calibration device was described for calibrating AFM cantilevers using a piezolever. It was also used for determining the nonlinear AFM stage behavior. The stiffness of 13 AFM cantilevers were then measured using the technique. When appropriate, a comparison was made with results found using nanoindentation. The piezolever method has advantages over other techniques such as being fast, nondestructive, applicable to arbitrarily shaped and coated cantilevers, and is easy to implement due to its compact design. Most importantly, it provides a direct measure of the cantilever response in contrast to indirect calibration methods. The piezolever method can be applied to any commercially available AFM with no additional equipment. In addition, this method can be implemented without calibration of the AFM laser. It can easily be used at the start of each AFM measurement. The choice of piezolever dictates the range of cantilever stiffnesses that can be accurately assessed. For the piezolever used here, the maximum stiffness is $6.02 \mathrm{~N} / \mathrm{m}$ while the lower limit of stiffness is estimated to be $0.08 \mathrm{~N} / \mathrm{m}$.

\section{ACKNOWLEDGMENTS}

The authors wish to thank Hiroshi Takahashi (Seiko Instruments Inc.) for his valuable help and technical support. The support of the U.S. National Science Foundation (Grant Nos. DMI-0210850 and EPS-0346776) and the Army Research Laboratory (RMAC-RTP Cooperative Agreement No. W911NF-04-2-0011) is also gratefully acknowledged.

${ }^{1}$ G. Binnig, C. F. Quate, and C. Gerber, Phys. Rev. Lett. 56, 930 (1986).

${ }^{2}$ D. Sarid, Scanning Force Microscopy: With Applications to Electric, Magnetic, and Atomic Forces, 2nd ed. (Oxford University Press, New York, 1994).

${ }^{3}$ C. T. Gibson, G. S. Watson, and S. Myhra, Nanotechnology 7, 259 (1996).

${ }^{4}$ B. Cappella and G. Dietler, Surf. Sci. Rep. 34, 1 (1999).

${ }^{5}$ H. Clausen-Schaumann, M. Rief, C. Tolksdorf, and H. E. Gaub, Biophys. J. 78, 1997 (2000).

${ }^{6}$ L. K. Tamm and Z. Shao, Biomembrane Structure (IOS Press, Amsterdam, 1998), pp. 169-185.

${ }^{7}$ V. J. Morris, A. R. Kirby, and A. P. Gunning, Atomic Force Microscopy for Biologists (Imperial College Press, London, 1999).

${ }^{8}$ M. Radmacher, Methods Cell Biol. 68, 67 (2002).

${ }^{9}$ H. Li, A. F. Oberhauser, S. B. Fowler, J. Clarke, and J. M. Fernandez, Proc. Natl. Acad. Sci. U.S.A. 97, 6527 (2000).

${ }^{10}$ M. Kudera, C. Eschbaumer, H. E. Gaub, and U. S. Schubert, Adv. Funct. Mater. 13, 615 (2003).

${ }^{11}$ T. Akiyama, U. Staufer, N. Rooij, L. Howald, and L. Scandella, Microelectron. Eng. 57-58, 769 (2001).

${ }^{12}$ Y. Miyahara, T. Fujii, S. Watanabe, A. Tonoli, S. Carabelli, H. Yamada, and H. Bleuler, Appl. Surf. Sci. 140, 428 (1999).

${ }^{13}$ T. Hantschel, S. Slesazeck, P. Niedermann, P. Eyben, and W. Vandervorst, Microelectron. Eng. 57-58, 749 (2001).

${ }^{14}$ T. Itoh and T. Suga, Sens. Actuators, A 54, 477 (1996).

${ }^{15}$ W. Kulisch, A. Malave, W. Scholz, C. Mihalcea, E. Oesterschulze, and G. Lippold, Diamond Relat. Mater. 6, 906 (1997).

${ }^{16}$ D. Sarid, Comput. Mater. Sci. 5, 291 (1996).

${ }^{17}$ K. Unno, T. Shibata, and E. Makino, Sens. Actuators, A 88, 247 (2001).

${ }^{18}$ G. Y. Chen, R. J. Warmack, A. Huang, and T. Thundat, J. Appl. Phys. 78, 1465 (1995).

${ }^{19}$ J. E. Sader, Technical Report, Encyclopedia of Surface and Colloid Science.

${ }^{20}$ J. E. Sader, Rev. Sci. Instrum. 66, 4583 (1995).

${ }^{21}$ J. M. Neumeister and W. A. Ducker, Rev. Sci. Instrum. 65, 2527 (1994).

${ }^{22}$ U. D. Schwarz, P. Köster, and R. Wiesendanger, Rev. Sci. Instrum. 67, 2560 (1996).

${ }^{23}$ H. J. Butt, P. Siedle, K. Seifert, K. Fendler, T. Seeger, E. Bamberg, A. Weisenhorn, K. Goldie, and A. Engel, J. Microsc. 169, 75 (1993).

${ }^{24}$ T. J. Senden and W. A. Duckert, Langmuir 10, 1003 (1994).

${ }^{25}$ Y. Li, N. Tao, J. Pan, A. A. Garcia, and S. Lindsay, Langmuir 9, 637 (1993).

${ }^{26}$ Y. Rabinovich and R.-H. Yoon, Langmuir 10, 1903 (1994).

${ }^{27}$ A. Torii, M. Sasaki, K. Hane, and S. Okuma, Meas. Sci. Technol. 7, 179 (1996).

${ }^{28}$ M. Tortonese and M. Kirk, Int. Soc. Opt. Eng. 3009, 53 (1997).

${ }^{29}$ J. P. Cleveland, S. Manne, D. Bocek, and P. K. Hansma, Rev. Sci. Instrum. 64, 403 (1993).

${ }^{30}$ J. L. Hutter and J. Bechhoefer, Rev. Sci. Instrum. 64, 1868 (1993).

${ }^{31}$ J. E. Sader, I. Larson, P. Mulvaney, and L. R. White, Rev. Sci. Instrum. 66, 3789 (1995) 
${ }^{32}$ J. E. Sader, J. W. M. Chon, and P. Mulvaney, Rev. Sci. Instrum. 70, 3967 (1999).

${ }^{33}$ J. D. Holbery, V. L. Eden, M. Sarikaya, and R. M. Fisher, Rev. Sci. Instrum. 71, 3769 (2000).

${ }^{34}$ I. Behrens, L. Doering, and E. Peiner, J. Micromech. Microeng. 13, S171 (2003).

${ }^{35}$ J. R. Pratt, J. A. Kramar, D. B. Newell, and D. T. Smith, Meas. Sci. Technol. 16, 2129 (2005).
${ }^{36}$ P. J. Cumpson, P. Zhdan, and J. Hedley, Ultramicroscopy 100, 241 (2004).

${ }^{37}$ SII Nanotechnology Inc., Tsukiji Bldg. Shintomi 2-15-5, Chuo-Ku Tokyo 104-0041, Japan, http//:www.siint.com

${ }^{38}$ Oliver, W. C. and Pharr, G. M., J. Mater. Res. 7, 1564 (1992).

${ }^{39}$ Z. Hu, T. Seeley, S. Kossek, and T. Thundat, Rev. Sci. Instrum. 75, 400 (2004).

${ }^{40}$ Hysitron, 5251 West 73rd Street, Minneapolis, MN 55439.

${ }^{41}$ Micromasch, http://www.spmtips.com/nsc11/Si3n4/al-Bs 